\title{
CUANTIFICACIÓN Y DISTRIBUCIÓN CARTOGRÁFICA DE LA GENERACIÓN DE ESCORRENTÍA Y SEDIMENTOS EN LA PROVINCIA DE MÁLAGA
}

\author{
Antonio Gallegos Reina
}

\begin{abstract}
RESUMEN
El artículo realiza una aproximación a los riesgos de inundación y erosión en la provincia de Málaga, desde la cuantificación y distribución cartográfica de los procesos de generación de escorrentía y sedimentos. Para estimar la generación de escorrentía se ha trabajado con el umbral de escorrentía y con las máximas lluvias diarias. La generación de sedimentos se ha modelizado aplicando cartográficamente la Ecuación Universal de Pérdida de Suelos. Finalmente, se han calculado diversas estadísticas para cada una de estas variables tanto a nivel provincial como municipal. Paralelamente, durante el trabajo se incide en la interrelación entre las peligrosidades por inundabilidad y erosión, y la necesidad de estudiar ambos riesgos de manera asociada.
\end{abstract}

\begin{abstract}
The article is an approach to the risks of flooding and erosion in the province of Málaga, since the quantification and distribution mapping of runoff generation processes and sediments. To estimate runoff generation has worked with the runoff threshold and the maximum daily rainfall. Sediment generation was modeled by applying Universal Soil Loss Equation. Finally, various statistics were calculated for each of these variables at both the provincial and municipal levels. In parallel, during the work bearing on the interrelationship between the hazardousness by flooding and erosion, and the need to consider both risks together.
\end{abstract}

PALABRAS CLAVE: Inundación, erosión, umbral de escorrentía, sedimentos, Málaga

KEY WORDS: Flood, erosion, runoff threshold, sediments, Málaga

\section{RIESGOS NATURALES EN LA PROVINCIA DE MÁLAGA E INTE- RRELACIÓN ENTRE LA INUNDABILIDAD Y LA PRODUCCIÓN DE SEDIMENTOS}

Conociendo la cantidad y el modo en que se generan la escorrentía y los sedimentos por erosión hídrica en una región determinada, puede obtenerse una primera aproximación cuantitativa y distributiva de la peligrosidad por inundación, además de la propia peligrosidad por erosión de suelos y los procesos asociados entre ambas. 
Inundabilidad y erosión de suelos son los riesgos naturales más importantes, en términos sociales y económicos, de la provincia de Málaga. Es por ello de capital importancia su estudio para la incorporación de conclusiones y propuestas de acción al planeamiento territorial y urbanístico provincial o municipal.

En relación al riesgo de inundabilidad, en la actualidad solo el primer factor, la generación de escorrentía, se incorpora a los estudios hidrológicohidráulicos que se presentan a las administraciones competentes, si bien existe una estrecha interrelación entre la producción de sedimentos y la inundabilidad, tanto por su acción como factor agravante como al actuar de desencadenante de nuevos procesos.

Esta relación es especialmente notable en nuestra provincia, donde la torrencialidad de su clima llega a producir y evacuar grandes cantidades de sedimentos en periodos de tiempo muy cortos, con lo que el flujo de escorrentía lleva una importante proporción de carga sólida y alta competencia (figura 1), además de aterrar cauces e infraestructuras o desencadenar deslizamientos asociados a la inundación.
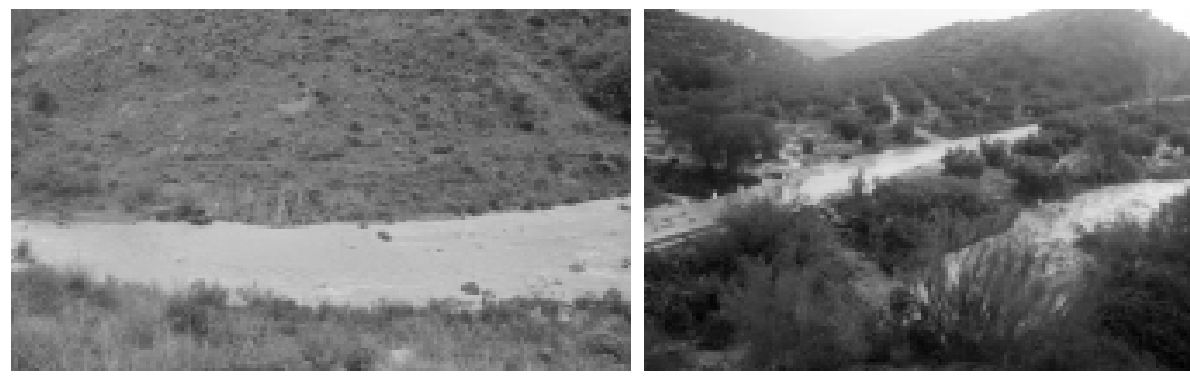

Figura 1. Fotografías de detalle de crecidas, en las que se aprecia que la carga de suelo arrastrada por el flujo transforma el caudal en una mezcla densa de barro con importante porcentaje de partículas sólidas.

Desde un punto de vista normativo, con la directiva 2007/60/CE, relativa a la evacuación y gestión de los riesgos de inundación, se avanza en los requerimientos para unificar riesgos con funcionamientos y consecuencias asociados, de modo que en su artículo 6.5.d se cita la necesidad de incorporar a la cartografía de inundabilidad la existencia de "aquellas zonas que puedan producir un alto contenido de sedimentos y flujos de derrubios" (Perles 2010).

Caben citar también experiencias avanzadas al respecto a nivel nacional, como la planificación de espacios fluviales de Cataluña (PEFCAT), que divide su análisis en tres aspectos: el estudio hidrológico-hidráulico, el ecológico y el morfodinámico, atendiendo en éste último al análisis de la erosión y la generación y transporte de sedimentos (Borras 2006). 


\section{CARTOGRAFÍA DE GENERACIÓN DE ESCORRENTÍA}

La provincia de Málaga se caracteriza por reunir algunos de los principales factores determinantes del riesgo de inundación. Entre estos, destacan la vulnerabilidad y exposición de su territorio, la torrencialidad de las precipitaciones, el bajo umbral de escorrentía y el tamaño y forma de las cuencas.

Centrándonos en la peligrosidad y obviando el análisis local de las cuencas, se puede obtener un mapa de peligrosidad provincial atendiendo a la capacidad de infiltración de los suelos y a la probabilidad estadística de precipitaciones extremas. Cada uno de estos factores se ha calculado como mapas independientes que luego se han unido en el mapa final de generación de escorrentía.

\subsection{Cartografía de umbrales de escorrentía o capacidad de infiltración de suelos}

El umbral de escorrentía se ha obtenido a partir del método del número de curva, del Soil Conservation Service (USSCS 1972). El método, modificado y adaptado para el caso español por Témez $(1978,1991)$, es ampliamente utilizado por la facilidad para estimar sus parámetros a partir de criterios fácilmente evaluables, como la pendiente, los usos del suelo, sus características hidrológicas o una clasificación basada en la potencia, textura y drenaje del suelo, y que se ha sintetizado a partir de la litología.

De la unión de todo ello se obtiene un umbral inicial de escorrentía, que define a partir de qué momento el suelo deja de infiltrar y comienza a producir escorrentía.

Para la incorporación en un Sistema de Información Geográfica de las variables citadas se han elaborado previamente mapas de pendientes, geología y usos del suelo de toda la provincia, clasificando cada una de ellos en los intervalos que se ajustan a las tablas consideradas en el método. Una vez obtenido un valor individual para cada combinación posible, se ha traído a una hoja de cálculo que decodifica el umbral correspondiente.

Los mapas citados se homogeneizaron a una escala 1/10.000, y se han rasterizado con un tamaño de celda de 10x10 metros, lo que supone una resolución suficiente ya no solo para usar sus datos a nivel provincial, sino también para ámbitos municipales o inferiores.

$\mathrm{Al}$ valor resultante posteriormente se le ha aplicado un coeficiente corrector que refleje la variación regional de la humedad habitual en el suelo al comienzo de los aguaceros.

Con ello, finalmente, y para cada celda, obtenemos el umbral, en litros por metro cuadrado, a partir del cual, un evento pluviométrico comienza a generar escorrentía (figura 2). 
Del resultado de este mapa se desprende un valor de umbral medio provincial de 70,5 mm. Los municipios con menor capacidad de infiltración son los axárquicos. En éstos, y como término medio, los suelos convierten en escorrentías lluvias superiores a $47 \mathrm{1} / \mathrm{m}^{2}$. Otros municipios con problemática similar son Fuengirola, Benalmádena, Rincón de la Victoria o Torremolinos, donde lluvias inferiores a $40 \mathrm{l} / \mathrm{m}^{2}$ ya generan escorrentía.

Por el contrario, los suelos más aptos para infiltrar las precipitaciones son los de la Serranía de Ronda, donde la media es de $90,3 \mathrm{l} / \mathrm{m}^{2}$.

Esta distribución de los valores finales responden a la división de grupos hidrológicos de suelos en la provincia, con suelos excesivamente drenados, formados fundamentalmente por materiales arenosos o areno-limosos, en el alto y medio Valle del Guadalhorce y determinados valles del litoral occidental; suelos moderadamente drenados en el bajo Valle del Guadalhorce, llanura antequerana y buena parte de las serranías occidentales de la provincia; suelos imperfectamente drenados, de materiales esquistosos y marmóreos, en la comarca de la Axarquía, Valle del Genal y entorno de la Sierra de Mijas; y suelos pobremente drenados, fundamentalmente arcillas y margas, en las laderas meridionales del cinturón serrano calizo y valles del Guadalteba y Turón.

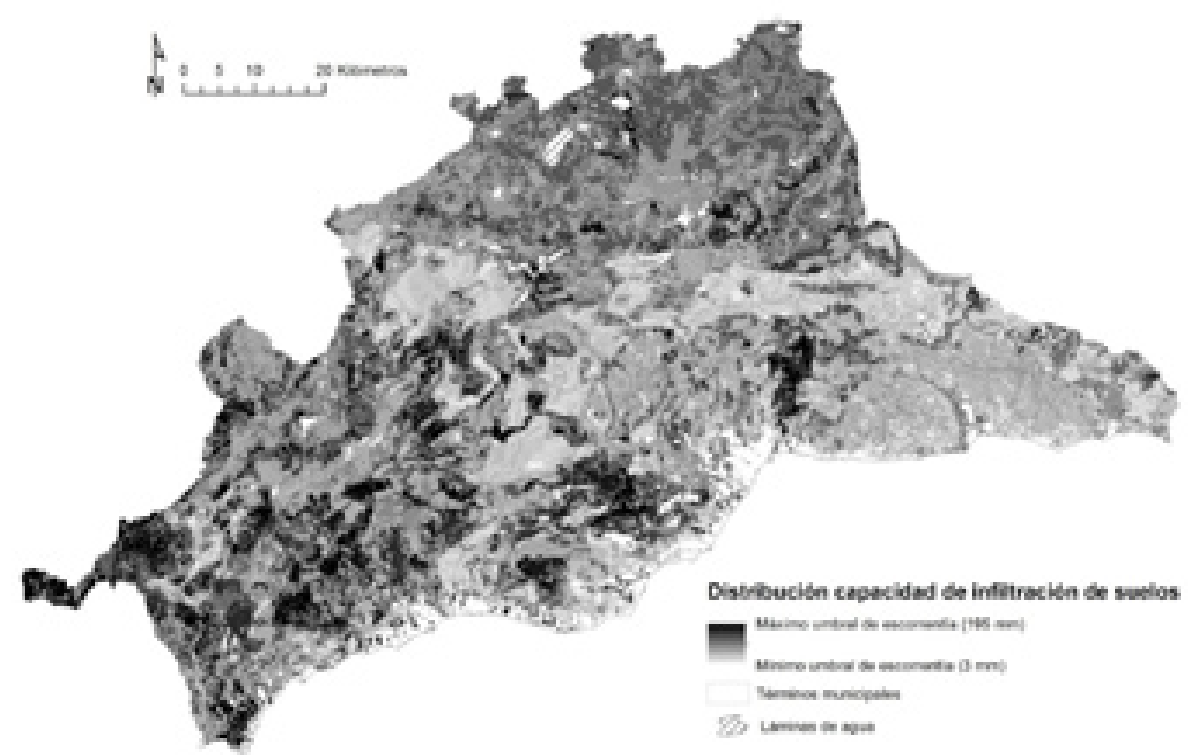

Figura 2. Cartografía de distribución de la capacidad de infiltración de suelos, medida en litros $/ \mathrm{m}^{2}$, en la provincia de Málaga para el año 2003. 
Responden, de igual modo, a las pendientes y a los usos del suelo y sus características hidrológicas, con las principales masas forestales de la provincia en las serranías rondeña, de Tejeda y Almijara, del cinturón calizo y de los Montes de Málaga, donde se contrarrestan la capacidad de retención de los suelos forestales con la mayor pendiente de estas regiones. Tiene mayor peso de cara a la infiltración, no obstante, el primero de los factores, el uso del suelo.

\subsection{Cartografía de máximas lluvias diarias o lluvias de carácter torrencial}

Junto al umbral de escorrentía, se ha realizado el mapa de máximas lluvias diarias de la provincia, con el que se pretenden evaluar de manera zonal aquellas lluvias de carácter torrencial que puedan dar lugar a inundaciones u otros riesgos naturales asociados. Este dato es la media del día con mayor precipitación anual de toda la serie, y con ello se filtrará el mapa de precipitaciones únicamente a aquellas que puedan originar peligrosidad.

Este mapa se ha obtenido a partir de los datos estadísticos de 140 estaciones meteorológicas de la provincia de Málaga, aportados por la Agencia Estatal de Meteorología, seleccionando la información disponible para trabajar únicamente con estaciones que tengan un registro mínimo de 20 años completos. Los datos se han interpolado en el Sistema de Información Geográfica mediante el método de krigeado, que pondera los valores medidos circundantes para calcular la predicción de ubicaciones sin medición, estimando con ello la variación espacial continua que se presume en el comportamiento de las precipitaciones.

De manera homogénea a la anterior cartografía, el tamaño de celda con el que se ha calculado el ráster resultante es de 10x10 metros.

De este segundo mapa (figura 3) se desprende que el riesgo de precipitaciones intensas es mayor en la franja formada por la banda montañosa de la mitad occidental de la provincia (sierras costeras occidentales y serranía de Ronda) y en el conjunto de las sierras Tejeda y Almijara, junto con los Montes de Málaga. En toda esta región la precipitación media máxima diaria anual oscila entre 70 y $120 \mathrm{l} / \mathrm{m}^{2}$.

Los valores de precipitación extrema más bajos se localizan en la comarca de Antequera.

El valor medio para la provincia es de $66,61 / \mathrm{m}^{2}$. 


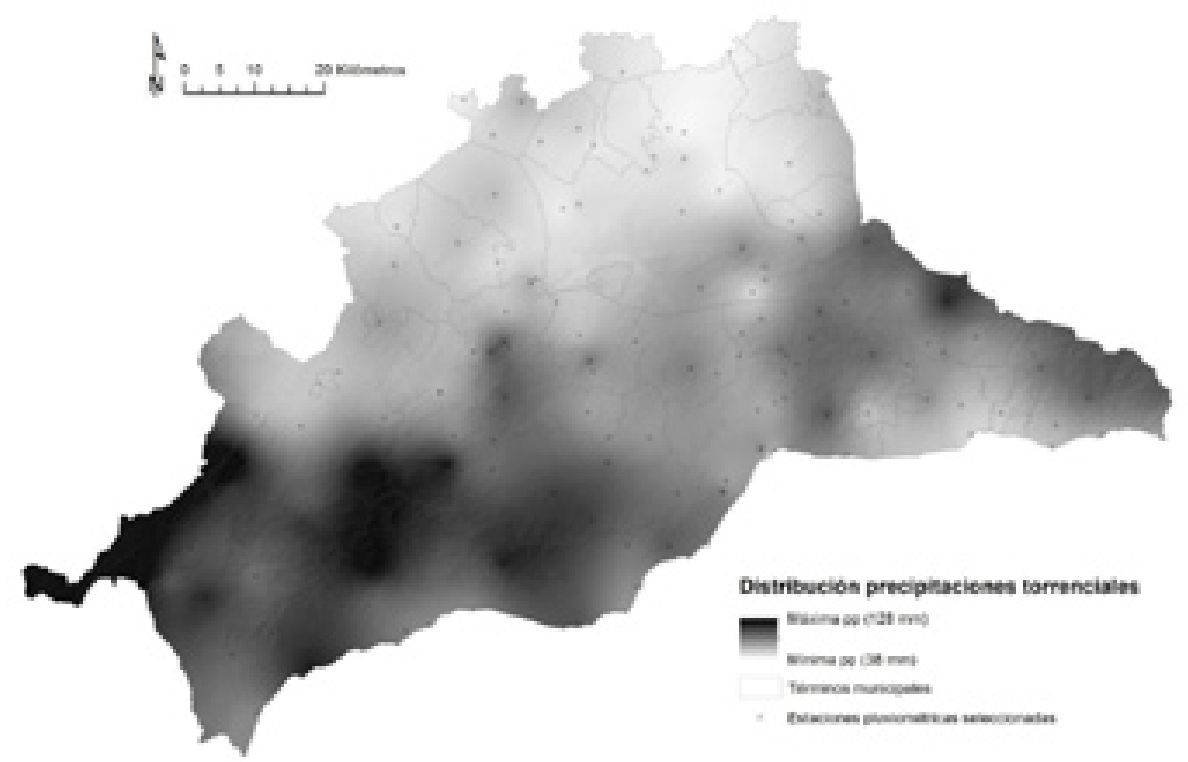

Figura 3. Cartografía de distribución de precipitaciones torrenciales (media de las máximas lluvias diarias anuales), medida en litros $/ \mathrm{m}^{2}$, en la provincia de Málaga.

\subsection{Cartografía final de generación de escorrentía en eventos torrenciales}

El proceso de generación de escorrentía viene determinado, de un lado, por la cuenca de drenaje, atendiendo a su configuración hidrogeomorfológica, cubierta de suelo y humedad antecedente, y de otro lado por la lluvia, considerada según volumen, intensidad y distribución espacio-temporal (Camarasa, 2004). En atención a esto, y resultado de la unión de los mapas de umbral de escorrentía y distribución de precipitaciones máximas, obtenemos un valor medio de escorrentía para eventos tormentosos extremos de periodicidad anual.

Conociendo la precipitación máxima anual esperada en 24 horas y el umbral de escorrentía en cada celda de trabajo, se obtiene en litros $/ \mathrm{m}^{2}$ la escorrentía esperada en cada píxel de trabajo (figura 4).

Analizando esta cartografía observamos el modo en que se distribuye la probabilidad de que se produzcan escorrentías elevadas, y en una primera aproximación, también el mayor riesgo de inundación. Los valores más altos se localizan en los municipios de la Axarquía y del litoral occidental, apareciendo seguidamente otros municipios serranos como Guaro, Monda, Benaoján o Coín. 


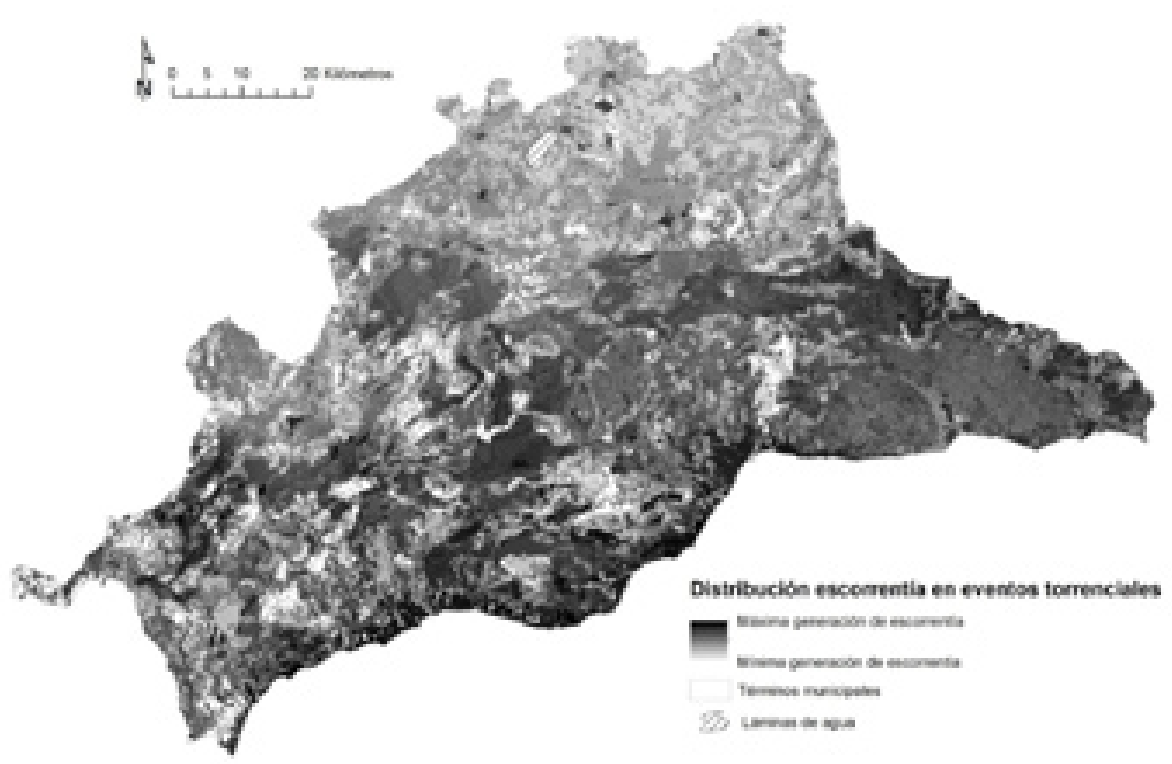

Figura 4. Cartografía de distribución de la generación de escorrentía, medida en litros $/ \mathrm{m}^{2}$, en eventos torrenciales en la provincia de Málaga.

\section{DISTRIBUCIÓN DE LA GENERACIÓN DE SEDIMENTOS EN LA PROVINCIA DE MÁLAGA}

La generación de sedimentos se estudiará mediante el cálculo de erosión potencial de suelos, que nos dará valores de toneladas métricas de suelo por hectárea y año.

La pérdida de suelo tiene una significativa relación con los objetivos de este trabajo, en su doble vertiente de riesgo natural en sí mismo y de incorporación de sólidos a la escorrentía y flujo de los cauces, y con ello, al incremento de la peligrosidad de la inundabilidad.

No obstante, la relación entre esta generación de sedimentos y la inundación va más allá del mero incremento de peligrosidad al aumentar la densidad de su flujo. El aporte de estos sólidos al caudal aumenta la energía erosiva en el propio cauce, especialmente en las márgenes cóncavas de los meandros, propiciando con ello el desbordamiento en estos puntos. Esto, a su vez, redunda en el aumento de acarreos procedentes de la erosión del cauce, de modo que el proceso se retroalimenta. Llegado a cierto punto, se puede producir el zapamiento de la base de la ladera, lo que da lugar a deslizamientos de ésta.

De igual modo, el aporte de sólidos limita la capacidad de evacuación del cauce, y en determinados casos incluso lo colmata. Con ello se pierde capa- 
cidad de evacuación del caudal, desbordamiento y expansión de la lámina de agua. Asimismo, esta expansión propicia movimientos gravitacionales en laderas que sean susceptibles a ello y que se vean afectadas por la inundación.

Por otro lado, en estrechamientos naturales o artificiales del cauce se pueden producir colapsos del drenaje y represamiento temporal, que al resolverse mediante rotura por incremento de la presión del flujo represado, generan un picos de crecida muy destructivos (Perles 2006).

Junto a lo anterior, debemos tener presente que algunas de las comarcas de nuestra provincia tienen unas condiciones climáticas regionales de carácter semiárido, en las que el fenómeno erosivo derivará en un proceso de desertificación del territorio, con graves repercusiones ya no solo en los biotopos pre-existentes, sino también en el aprovechamiento agrícola o económico del territorio.

\subsection{Ecuación universal de pérdida de suelo revisada}

La erosión potencial o erosionabilidad hace referencia a la susceptibilidad a la erosión que se prevé va a tener lugar en el futuro bajo unas características geográficas y antrópicas determinadas. No se mide directamente, sino que se obtiene a partir de las variables que van a condicionar el proceso erosivo.

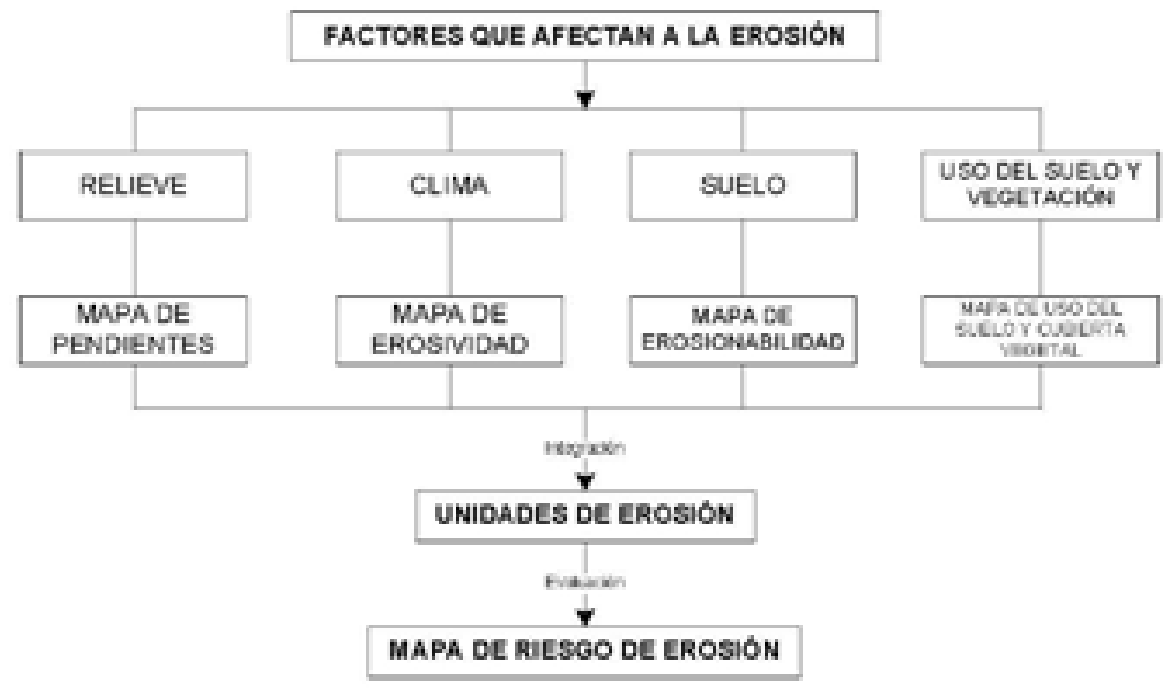

Figura 5. Esquema metodológico para la definición de paisajes erosivos (ICONA, 1982) 
La cuenca mediterránea, y la región que nos ocupan en particular, se caracterizan por la coincidencia de los periodos de sequía con los eventos de precipitaciones torrenciales, que se convierte con ello en el principal agente erosivo y causante prioritario del arrastre del mayor volumen de sedimentos. El análisis de la generación de sedimentos se hará por ello mediante un modelo de erosión hídrica. El método de más difusión es la ecuación universal de pérdida de suelo revisada (RUSLE).

Cabe tener presente que la calibración de esta ecuación para el área mediterránea podría tener sensibles inadaptaciones, a pesar de lo cual se tomará por su valor comparativo y estimativo, dado que la búsqueda de otra opción nos obligaría al uso de parcelas experimentales, imposibilitando la realización de estudios regionales de cierta extensión, y tanto menos provinciales.

La ecuación universal de pérdida de suelo modificada (Wischmeier 1958) y su revisión descrita por Mannaerts (1999), conocida como RUSLE, responde a la siguiente expresión matemática:

$A=R \times K \times L \times S \times C \times P$

Ecuación 1. Pérdida de suelo promedio anual según USLE revisada (RUSLE)

$\mathrm{A}=$ pérdida de suelo media anual por unidad de superficie $\left(\mathrm{T}_{\mathrm{m}} \cdot \mathrm{ha}^{-1} \cdot \mathrm{año}^{-1}\right)$

$\mathrm{R}=$ factor de erosividad de las lluvias $\left(\mathrm{KJ} \cdot \mathrm{mm} \cdot \mathrm{m}^{-2} \cdot\right.$ hora $\left.^{-1} \cdot \mathrm{año}^{-1}\right)$

$\mathrm{K}=$ factor de erodabilidad del suelo $\left(\mathrm{T}_{\mathrm{m}} \cdot \mathrm{m}^{2} \cdot\right.$ hora $\left.\cdot \mathrm{ha}^{-1} \cdot \mathrm{KJ}^{-1} \cdot \mathrm{mm}^{-1}\right)$

$\mathrm{L}=$ factor longitud de pendiente (adimensional)

$\mathrm{S}=$ pendiente $(\%)$ (adimensional)

$\mathrm{C}=$ factor de cubierta vegetal (adimensional, tabulado según las características de la vegetación)

$\mathrm{P}=$ factor de prácticas de conservación de cultivo (adimensional)

Cada factor responde a lo siguiente:

Erosividad de la lluvia (Factor R):

El factor $R$ se define como el índice medio anual de la erosividad de la lluvia. Existen diversos métodos de cálculo, siendo el más usado el análisis de las precipitaciones medias mensuales máximas y medias anuales.

Así, el cálculo de " $R$ " se ha efectuado mediante la ecuación de Lombardi Neto y Moldenhauer (1.980), mediante los mapas de precipitación media anual y precipitación media mensual máxima de la provincia de Málaga. 


\section{Erodabilidad del suelo (factor $K$ ):}

Este factor expresa la influencia de las propiedades químicas y físicas del suelo en la erosión, a través de la infiltración, permeabilidad, capacidad de retención de agua, resistencia a la dispersión, aplastamiento, abrasión y fuerzas de transporte. Indica, en resumen, la susceptibilidad de un suelo para ser erosionado.

El resultado es un valor de cantidad de suelo perdido, asumiendo unas condiciones estándar de pendiente del $9 \%$, longitud de $22,13 \mathrm{~m}$ y en barbecho continuo.

Generalmente son menos erosionables los suelos profundos permeables de arenas gruesas. Los más erosionables serán los suelos arenosos y poco profundos sobre subsuelo impermeable.

Para facilitar el cálculo de $K$, Wishcmele et al. (1971) han elaborado un nomograma que resuelve gráficamente la ecuación que liga las variables del proceso de erosión. A partir de la relación porcentaje de limo y arcillas, materia orgánica y permeabilidad podemos extraer el valor de $K$, que luego a su vez ha sido sintetizado y adaptado a la geología regional.

Factor topográfico $(L \cdot S)$ :

El factor $L$, o longitud del declive, viene definido una formulación matemática que incluye la longitud del declive, o distancia desde el talweg hasta el interfluvio, y un exponente influenciado principalmente por la interacción entre la longitud de declive y la pendiente.

El factor $S$, o factor de inclinación de la pendiente, responde a otra formulación matemática basada en la pendiente.

Ambas variables suelen agruparse en una tercera formulación, bajo la denominación de factor topográfico $L \cdot S$, como factor que integra el efecto del relieve, longitud del declive o ladera y pendiente. Existen dos ecuaciones diferentes en función de la pendiente y longitud de declive.

Factor cubierta vegetal $(C)$ :

Este factor medirá la influencia de la cobertura vegetal sobre el terreno, lo que resulta especialmente relevante al ser el medio más eficaz para que la erosión disminuya. El factor $C$ está basado en el concepto de desviación estándar, siendo el estándar un área bajo condiciones de barbecho con cultivo limpio en cuyo caso su valor es 1 .

Inicialmente con este factor se consideró el manejo del cultivo, pero por extensión, para suelos no agrícolas, se ha asimilado al valor de protección ejercido por la cubierta vegetal, con cuyo porcentaje de cobertura del suelo se relaciona de manera inversa la tasa de erosión.

Su valor varía entre 0 y 1 y disminuye a medida que aumenta la cobertura vegetal. 
Este factor es determinante en el flujo de retención del suelo, y su valor aparece en el modelo como constante, introducido manualmente a partir de una tabla propuesta por el ICONA. Esto llevó a que, en el planteamiento de los estudios del proyecto LUCDEME, cuyos resultados fueron publicados por ICONA en 1982, el factor $C$ fuese analizado igualmente de forma simplificada. De este modo, los diferentes tipos de vegetación cartografiados en el Mapa de Cultivos y Aprovechamientos, realizado por el Ministerio de Agricultura, fueron agrupados y valorados en una serie de tipos de cubiertas que han sido usadas en este trabajo.

Estos valores reflejan como las zonas de vegetación natural, en la cual existe una cierta potencia en el desarrollo de la misma, los suelos están bastante protegidos porque suelen tener una densa vegetación en la parte aérea (copas) y en la parte subterránea (raíces), disminuyendo con ello la energía cinética de la lluvia y evitando la desestructuración del suelo.

Factor de prácticas de conservación de suelo $(P)$ :

El factor de prácticas de conservación de suelos expresa la influencia que ejercen las prácticas de cultivo, corrección y conservación de la erosión hídrica. La función de este valor es disminuir el valor de pérdida de suelo obtenido, suponiendo que existen o van a llevarse a cabo prácticas de conservación, tales como cultivos en fajas, aterrazados, etc. en el área de estudio.

Se denominan también prácticas de contorno, e incluye cualquier tipo de obra, barrera vegetal o incluso la propia disposición de las especies vegetales plantadas o repobladas.

Algunas de estas prácticas de conservación de suelos pueden ser:

Aterrazamientos en zonas de montaña, buscando disminuir la pendiente, favorecer la infiltración y reducir la velocidad de la escorrentía.

Alternar especies poco densas con especies más densas en laderas de gran longitud.

Cuando no existe ninguna medida de protección el valor Standard de $P$ es igual a 1 . A medida que se practican medidas de contorno el valor de $P$ actúa como un atenuador del valor final de la erosión.

\subsection{Proceso de elaboración cartográfica}

Para la elaboración del mapa se ha trabajado con un total de 7.453 cuencas fluviales, que cubren la totalidad de la superficie provincial (figura 6). Estas cuencas se han obtenido mediante un proceso automático gracias al uso de las Tecnologías de la Información Geográfica, usando un modelo digital del terreno calculado tras reunir todas las curvas de nivel provinciales a escala $1 / 10.000$. 


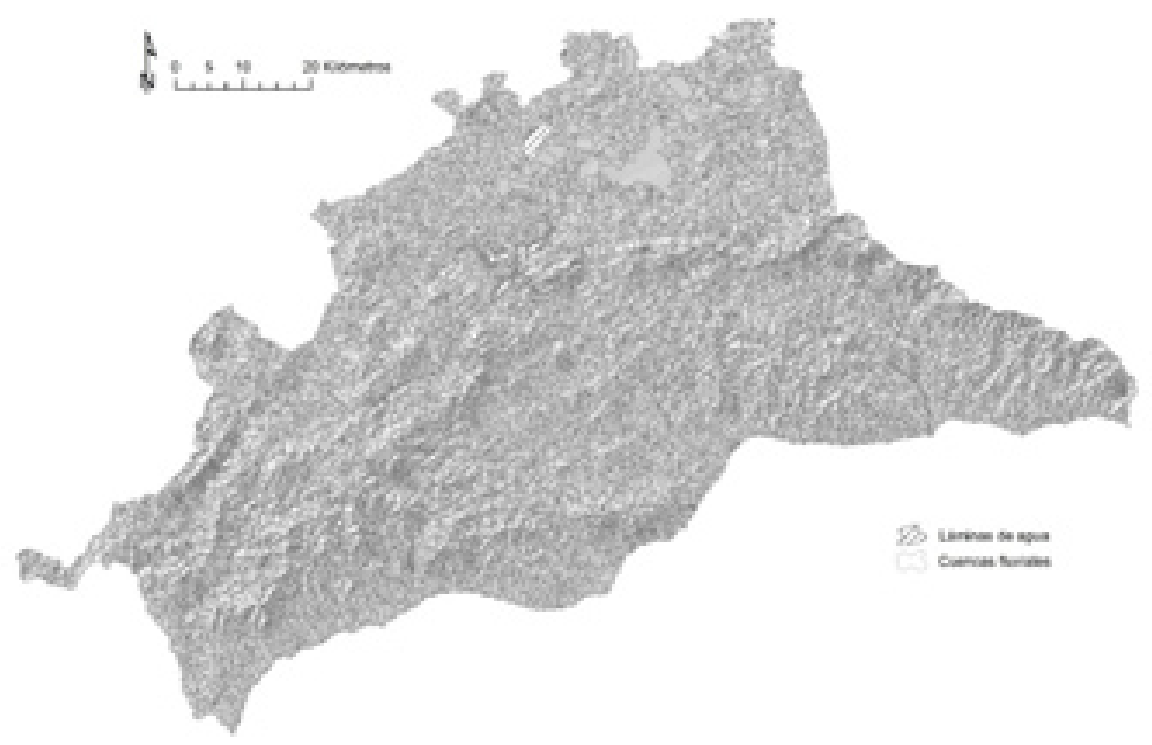

Figura 6. Cuencas fluviales usadas para los cálculos del factor topográfico.

Los pasos de este proceso han consistido en el reacondicionamiento del modelo digital, el relleno de depresiones o pozas, la generación del modelo de direcciones del flujo, la generación del modelo de acumulación de flujo, la definición automática de las líneas de corriente, la segmentación de éstas en divisorias hidráulicas, y por último la definición de las cuencas fluviales.

Una vez obtenida esta información y vectorizada, se han aplicado individualmente en cada una de las cuencas las variables desarrolladas en el apartado anterior, obtenidas a partir de cartografía provincial, elaborada o no, de geología, altimetría, morfometría de laderas, erosividad de la lluvia, usos del suelo o cubiertas vegetales.

Para el factor $L S$, por su complejidad matemática, ha sido necesario obtener los valores mediante hoja de cálculo, exportando los resultados a la cartografía en polígonos (subcuencas) de valores homogéneos.

La cartografía resultante final, con el valor de la erosión potencial medida en toneladas por hectárea y año, ha sido rasterizada, para favorecer los estudios estadísticos zonales, con celdas de 10x10 metros (figura 7).

\subsection{Resultados}

La erosión potencial de la provincia supera los 42 millones de toneladas métricas anuales, de lo que resulta una media superior a las 56 toneladas métricas de suelo perdido por hectárea y año. 
Un 50,5\% de la superficie provincial tiene una erosión potencial alta o muy alta, y ésta es especialmente intensa en la Axarquía-Montes de Málaga, en las laderas del entorno del Valle del Guadalhorce, en la fachada litoral occidental y en la comarca nororiental de Málaga.

El municipio con mayor erosión potencial es Benalmádena, con valores superiores a 130 toneladas por hectárea y año, mientras que en el extremo contrario se sitúan municipios de la Serranía de Ronda, cuya pérdida de suelos llega a bajar de las 20 toneladas por hectárea y año.

Esta distribución se justifica fundamentalmente por condicionantes como la mayor desprotección forestal de Axarquía y otras zonas agrícolas abandonadas de los Montes de Málaga, o la existencia de geologías más susceptibles a la erosión en las calizas subbéticas de la comarca nororiental de la provincia. Otros condicionantes negativos, pero con un menor peso relativo a la hora de configurar la distribución de la erosión potencial provincial, son la morfometría de las cuencas o el factor de erosividad de la lluvia, que es más desfavorable en la serranía rondeña o vega antequerana.

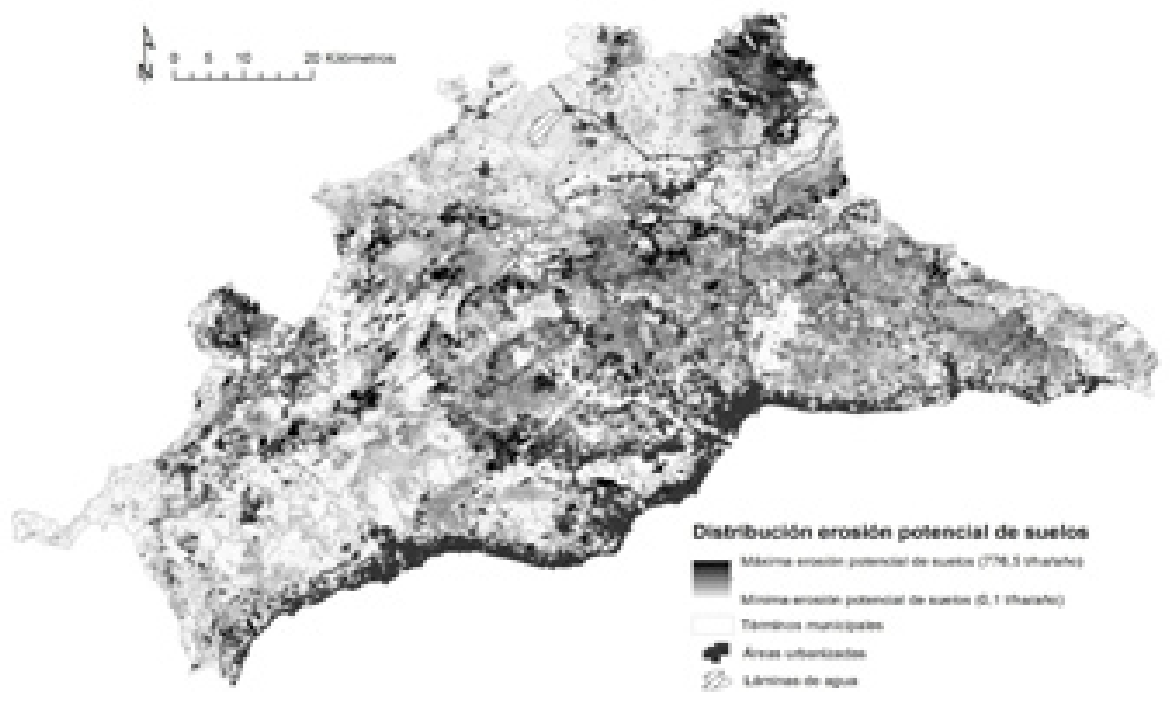

Figura 7. Cartografía de distribución de la producción de sedimentos o erosión potencial de suelo, medida en toneladas por hectárea y año, en la provincia de Málaga

\section{REFLEXIONES FINALES}

En los últimos años, el importante incremento de producción cartográfica digitalizada y el aumento de potencialidades de los Sistemas de Información 
Geográfica, han permitido ya no solo un uso extendido y frecuente de éstos, sino también su aplicación para realizar análisis matemáticos y cartográficos demasiado complejos hasta no hace mucho. Así, ha resultado posible trabajar aquí con una superficie de $7.306 \mathrm{~km}^{2}$ usando cartografía a escala 1/10.000, y aplicar operaciones matemáticas y cartográficas en más de 7.000 cuencas fluviales, con una superficie media inferior a las 100 hectáreas.

Es necesario tener presente que los resultados provienen de unas modelizaciones que pueden mostrar desajustes en el proceso en sí, en la adaptación de éstas a las peculiaridades geográficas de nuestra provincia y en las variables y escala consideradas para su funcionamiento.

Así, y por citar un ejemplo, en el cálculo de la cartografía de erosión potencial, se han tenido en cuenta los factores litológicos, topográficos, climáticos y biológicos, pero han quedado fuera factores como los estratigráficos y los estructurales, esto es, consideraciones como la alternancia de capas permeables/impermeables o la existencia de fallas, planos de cizallamiento o capas fuertemente inclinadas (Robredo 1993).

No obstante, sí debe darse por válido su valor estimativo, que a la luz de los resultados obtenidos no deja lugar a demasiadas dudas sobre el funcionamiento hidrológico y sedimentario de nuestras cuencas, y su valor comparativo para las distintas regiones o municipios provinciales.

Del análisis estadístico de estos resultados se desprende la necesidad de actuar de manera preventiva y urgente ante los elevados valores de escorrentía y erosión en la provincia de Málaga (ver tabla 1).

Se observa igualmente que los mayores ratios de las distintas peligrosidades consideradas se localizan en la franja litoral, coincidiendo de tal modo con la región de mayor exposición y vulnerabilidad de la provincia.

Las razones de las altas tasas de producción de escorrentía y sedimentos, más allá de los condicionantes climáticos y geomorfológicos apuntados en anteriores apartados, se originan en el desordenado y rápido desarrollo urbanístico que ha sufrido la provincia en las últimas décadas. El incremento de las zonas urbanas, y muy especialmente, las periurbanas y las urbanizaciones aisladas, junto con las infraestructuras que han traído aparejadas, como las viales, han supuesto una importante impermeabilización de cuencas y de desestructuración de suelos ya de por sí delgados y débilmente estructurados.

Esto produce más escorrentía y además, lo que circula no es agua limpia, sino una masa semi-sólida por el arrastre del suelo perdido, que termina taponando estructuras, como puentes y entubamientos, y aumentando la sinergia de los distintos factores que interactúan en el funcionamiento normal de todo riesgo natural. 
TABLA 1

RESUMEN DE VALORES MEDIOS POR MUNICIPIO DE UMBRAL DE ESCORRENTÍA, MÁXIMA LLUVIA DIARIA Y EROSIÓN POTENCIAL, Y VALORES ABSOLUTOS DE EROSIÓN POTENCIAL

\begin{tabular}{|c|c|c|c|c|c|}
\hline \multirow[b]{2}{*}{ MUNICIPIO } & \multirow[b]{2}{*}{$\begin{array}{c}\text { Superficie } \\
\text { municipal } \\
\left(\mathrm{km}^{2}\right)\end{array}$} & \multirow[b]{2}{*}{$\begin{array}{c}\text { Umbral de } \\
\text { escorrentía } \\
\left(1 / \mathrm{m}^{2}\right)\end{array}$} & \multirow{2}{*}{$\begin{array}{c}\text { Máximas } \\
\text { lluvias } \\
\text { diarias } \\
\left(1 / \mathrm{m}^{2}\right)\end{array}$} & \multicolumn{2}{|c|}{ Erosión potencial } \\
\hline & & & & \begin{tabular}{|c|} 
Valor \\
medio \\
(t/ha/año)
\end{tabular} & $\begin{array}{c}\text { Total } \\
\text { municipal } \\
\text { (t/año) }\end{array}$ \\
\hline ALAMEDA & 62.2 & 84.4 & 44.5 & 48.6 & 273131 \\
\hline ALCAUCIN & 45.3 & 53.3 & 83.9 & 71.2 & 328172 \\
\hline ALFARNATE & 33.4 & 73.0 & 76.6 & 49.9 & 165179 \\
\hline ALFARNATEJO & 20.0 & 51.4 & 74.7 & 57.7 & 115937 \\
\hline ALGARROBO & 9.7 & 32.6 & 50.2 & 51.7 & 48536 \\
\hline ALGATOCIN & 20.0 & 73.6 & 81.8 & 41.9 & 84218 \\
\hline ALHAURIN DE LA TORRE & 82.2 & 78.8 & 73.3 & 60.7 & 506426 \\
\hline ALHAURIN EL GRANDE & 73.1 & 108.9 & 82.7 & 48.0 & 339035 \\
\hline ALMACHAR & 14.7 & 42.6 & 64.8 & 52.1 & 78139 \\
\hline ALMARGEN & 34.7 & 70.4 & 45.0 & 36.5 & 120767 \\
\hline ALMOGIA & 163.6 & 57.8 & 64.2 & 70.8 & 1160093 \\
\hline ALORA & 169.2 & 54.2 & 59.5 & 74.7 & 1263860 \\
\hline ALOZAINA & 33.9 & 67.9 & 75.0 & 72.1 & 244541 \\
\hline ALPANDEIRE & 31.2 & 86.6 & 84.0 & 62.7 & 199271 \\
\hline ANTEQUERA & 813.6 & 71.5 & 49.4 & 49.1 & 4046801 \\
\hline ARCHEZ & 5.0 & 50.7 & 68.5 & 78.3 & 39280 \\
\hline ARCHIDONA & 186.5 & 83.3 & 46.3 & 59.1 & 1101190 \\
\hline ARDALES & 106.1 & 60.4 & 57.6 & 71.0 & 755620 \\
\hline ARENAS & 26.3 & 43.8 & 58.8 & 74.6 & 195913 \\
\hline ARRIATE & 8.3 & 66.4 & 52.0 & 59.8 & 50178 \\
\hline ATAJATE & 11.0 & 83.9 & 87.0 & 64.4 & 71991 \\
\hline BENADALID & 19.9 & 83.5 & 86.3 & 45.0 & 89773 \\
\hline BENAHAVIS & 144.8 & 83.8 & 89.0 & 34.3 & 495926 \\
\hline BENALAURIA & 20.6 & 85.5 & 84.5 & 32.9 & 67955 \\
\hline BENALMADENA & 27.0 & 26.5 & 76.8 & 130.0 & 293267 \\
\hline BENAMARGOSA & 11.9 & 47.2 & 68.1 & 57.6 & 68931 \\
\hline BENAMOCARRIA & 5.6 & 38.1 & 63.8 & 46.6 & 27035 \\
\hline BENAOJAN & 31.9 & 72.2 & 90.2 & 73.1 & 230973 \\
\hline BENARRABA & 25.9 & 80.7 & 82.7 & 25.7 & 66610 \\
\hline BORGE (EL) & 24.0 & 45.0 & 67.1 & 57.7 & 140946 \\
\hline BURGO (EL) & 187.1 & 73.4 & 59.3 & 53.0 & 621239 \\
\hline CAÑETE LA REAL & 42.1 & 45.0 & 52.3 & 59.2 & 949485 \\
\hline CAMPILLOS & 32.8 & 62.8 & 48.6 & 26.4 & 489914 \\
\hline
\end{tabular}




\begin{tabular}{|c|c|c|c|c|c|}
\hline \multirow[b]{2}{*}{ MUNICIPIO } & \multirow[b]{2}{*}{$\begin{array}{c}\text { Superficie } \\
\text { municipal } \\
\left(\mathrm{km}^{2}\right)\end{array}$} & \multirow{2}{*}{$\begin{array}{c}\text { Umbral de } \\
\text { escorrentía } \\
\left(1 / \mathrm{m}^{2}\right)\end{array}$} & \multirow{2}{*}{\begin{tabular}{|c|} 
Máximas \\
lluvias \\
diarias \\
$\left(1 / \mathrm{m}^{2}\right)$ \\
\end{tabular}} & \multicolumn{2}{|c|}{ Erosión potencial } \\
\hline & & & & $\begin{array}{c}\text { Valor } \\
\text { medio } \\
\text { (t/ha/año) }\end{array}$ & $\begin{array}{c}\text { Total } \\
\text { municipal } \\
\text { (t/año) }\end{array}$ \\
\hline CANILLAS DE ACEITUNO & 163.8 & 56.3 & \begin{tabular}{|l|}
69.4 \\
\end{tabular} & 62.5 & 264696 \\
\hline CANILLAS DE ALBAIDA & 22.5 & 71.2 & 74.6 & 42.0 & 139644 \\
\hline CARRATRACA & 21.9 & 71.2 & 71.8 & 49.8 & 112461 \\
\hline CARTAJIMA & 105.4 & 64.1 & 79.4 & 79.0 & 176918 \\
\hline CARTAMA & 68.3 & 61.0 & 67.8 & 57.9 & 622160 \\
\hline CASABERMEJA & 113.4 & 64.4 & 62.2 & 65.7 & 455670 \\
\hline CASARABONELA & 161.5 & 83.9 & 73.9 & 58.7 & 669994 \\
\hline CASARES & 127.2 & 67.9 & 78.5 & 40.1 & 641518 \\
\hline COIN & 66.1 & 58.1 & 83.2 & 64.7 & 794930 \\
\hline COLMENAR & 24.9 & 64.7 & 72.0 & 72.9 & 481628 \\
\hline COMARES & 53.2 & 50.3 & 74.3 & 64.2 & 161236 \\
\hline COMPETA & 174.5 & 115.8 & 76.3 & 55.0 & 300333 \\
\hline CORTES DE LA FRONTERA & 15.4 & 82.8 & 105.9 & 32.4 & 543826 \\
\hline CUEVAS BAJAS & 34.8 & 86.1 & 40.5 & 102.4 & 132172 \\
\hline CUEVAS DE SAN MARCOS & 16.1 & 50.2 & 43.7 & 120.9 & 349596 \\
\hline CUEVAS DEL BECERRO & 19.8 & 42.1 & 60.2 & 70.9 & 114687 \\
\hline CUTAR & 116.6 & 90.3 & 70.9 & 53.6 & 108925 \\
\hline ESTEPONA & 136.6 & 75.8 & 86.2 & 42.5 & 563644 \\
\hline FARAJAN & 20.5 & 96.0 & 84.8 & 17.8 & 36650 \\
\hline FRIGILIANA & 40.6 & 54.0 & 76.3 & 36.9 & 149739 \\
\hline FUENGIROLA & 10.1 & 21.3 & 69.9 & 82.2 & 63545 \\
\hline FUENTE DE PIEDRA & 90.7 & 71.8 & 46.6 & 15.1 & 136334 \\
\hline GAUCIN & 97.6 & 97.5 & 87.7 & 32.3 & 303313 \\
\hline GENALGUACIL & 31.5 & 87.5 & 79.4 & 31.6 & 99499 \\
\hline GUARO & 22.4 & 54.4 & 79.9 & 99.9 & 228868 \\
\hline HUMILLADERO & 34.7 & 90.2 & 44.5 & 30.0 & 103574 \\
\hline IGUALEJA & 44.3 & 110.4 & 95.1 & 22.5 & 99599 \\
\hline ISTAN & 100.1 & 78.8 & 88.5 & 33.5 & 335445 \\
\hline IZNATE & 7.5 & 44.7 & 61.5 & 45.1 & 33974 \\
\hline JIMERA DE LIBAR & 27.0 & 84.6 & 93.3 & 62.5 & 169486 \\
\hline JUBRIQUE & 39.1 & 85.6 & 81.3 & 40.3 & 163851 \\
\hline JUZCAR & 33.3 & 89.2 & 86.3 & 36.9 & 122755 \\
\hline MACHARAVIAYA & 7.4 & 44.3 & 56.5 & 33.6 & 24716 \\
\hline MALAGA & 394.0 & 61.2 & 67.4 & 51.4 & 1991687 \\
\hline MANILVA & 34.5 & 95.9 & 69.5 & 66.7 & 213818 \\
\hline MARBELLA & 116.5 & 40.4 & 76.6 & 113.1 & 1061322 \\
\hline MIJAS & 147.9 & 46.5 & 78.0 & 89.2 & 1126998 \\
\hline MOCLINEJO & 14.5 & 42.6 & 56.9 & 46.4 & 69090 \\
\hline
\end{tabular}




\begin{tabular}{|c|c|c|c|c|c|}
\hline \multirow[b]{2}{*}{ MUNICIPIO } & \multirow[b]{2}{*}{$\begin{array}{c}\text { Superficie } \\
\text { municipal } \\
\left(\mathrm{km}^{2}\right)\end{array}$} & \multirow[b]{2}{*}{$\begin{array}{c}\text { Umbral de } \\
\text { escorrentía } \\
\left(1 / \mathrm{m}^{2}\right)\end{array}$} & \multirow{2}{*}{$\begin{array}{c}\text { Máximas } \\
\text { lluvias } \\
\text { diarias } \\
\left(1 / \mathrm{m}^{2}\right) \\
\end{array}$} & \multicolumn{2}{|c|}{ Erosión potencial } \\
\hline & & & & \begin{tabular}{|c|} 
Valor \\
medio \\
$(\mathrm{t} / \mathrm{ha} / \mathrm{año})$
\end{tabular} & $\begin{array}{c}\text { Total } \\
\text { municipal } \\
\text { (t/año) }\end{array}$ \\
\hline MOLLINA & 74.5 & 88.8 & 43.0 & 29.6 & 220632 \\
\hline MONDA & 57.7 & 69.9 & 87.3 & 59.4 & 329689 \\
\hline MONTEJAQUE & 45.1 & 99.3 & 83.7 & 45.2 & 200275 \\
\hline NERJA & 83.7 & 48.2 & 72.3 & 37.4 & 313982 \\
\hline OJEN & 85.7 & 73.5 & 87.0 & 72.2 & 319972 \\
\hline PARAUTA & 44.1 & 95.9 & 93.6 & 48.2 & 214379 \\
\hline PERIANA & 58.3 & 38.0 & 74.0 & 63.9 & 376528 \\
\hline PIZARRA & 63.3 & 53.3 & 64.6 & 53.3 & 338884 \\
\hline PUJERRA & 24.4 & 114.8 & 92.1 & 13.2 & 32282 \\
\hline RINCON DE LA VICTORIA & 28.0 & 34.5 & 60.3 & 62.9 & 159593 \\
\hline RIOGORDO & 40.4 & 49.1 & 71.6 & 73.5 & 296911 \\
\hline RONDA & 478.8 & 80.4 & 61.9 & 53.5 & 2547987 \\
\hline SALARES & 10.7 & 66.2 & 68.8 & 43.5 & 48051 \\
\hline SAYALONGA & 18.2 & 45.2 & 60.2 & 61.9 & 113969 \\
\hline SEDELLA & 31.2 & 46.0 & 67.1 & 47.6 & 149913 \\
\hline SIERRA DE YEGUAS & 84.0 & 85.7 & 48.9 & 26.1 & 199429 \\
\hline TEBA & 144.0 & 58.6 & 50.1 & 57.1 & 829122 \\
\hline TOLOX & 93.9 & 79.7 & 88.1 & 48.0 & 452494 \\
\hline TORREMOLINOS & 20.3 & 38.0 & 73.8 & 80.8 & 143355 \\
\hline TORROX & 50.7 & 38.1 & 60.9 & 56.1 & 271820 \\
\hline TOTALAN & 9.2 & 47.5 & 67.6 & 50.1 & 46918 \\
\hline VALLE DE ABDALAJIS & 21.3 & 52.3 & 51.9 & 92.5 & 197335 \\
\hline VELEZ-MALAGA & 156.6 & 39.8 & 56.2 & 43.3 & 640894 \\
\hline VIÑUELA & 75.0 & 87.3 & 68.8 & 51.8 & 143387 \\
\hline VILLANUEVADE ALGAIDAS & 16.7 & 84.4 & 45.7 & 103.5 & 846078 \\
\hline VILLANUEVA DE TAPIA & 44.5 & 84.9 & 52.8 & 112.6 & 164648 \\
\hline VILLANUEVA DEL ROSARIO & 59.3 & 82.9 & 55.3 & 59.0 & 263443 \\
\hline VILLANUEVA DEL TRABUCO & 27.5 & 40.8 & 55.3 & 61.6 & 367916 \\
\hline YUNQUERA & 57.7 & 88.4 & 72.6 & 51.1 & 296371 \\
\hline MEDIA PROVINCIAL & 7284.3 & 70.5 & 66.6 & 56.3 & 40368745 \\
\hline
\end{tabular}




\section{BIBLIOGRAFÍA}

BORRAS, G. ET AL. (2006): "Delimitación de zonas inundables en el ámbito autonómico: Cataluña”, en DÍEZ HERRERO, A., LIN HUERTA, L. y LLORENTE ISIDRO, M. (eds.): Mapas de peligrosidad de avenidas e inundaciones. Métodos, experiencias y aplicación. Publicaciones del Instituto Geológico y Minero de España. Serie: Medio Ambiente. Riesgos Geológicos, 7, Madrid, 73-88.

CAMARASA A.M.; LÓPEZ GARCÍA, M.J. y PASCUAL, J.A. (2004): Análisis mediante SIG de los parámetros de producción de escorrentía, Departamento de Geografía, Universidad de Valencia.

ICONA (1982): "Paisajes erosivos en el sureste español. Ensayo de metodología para el estudio de su cualificación y cuantificación”, MAPA, Proyecto Lucdeme, Madrid.

LOMBARDI NETO, F. y MOLDENHAUER, W.C. (1980): "Erosividade da chuva: sua distribuiçaco e relação com perdas de solo em Campinas", en Encontro Nacional de Pesquisa sobre Conservação do Solo, Recife.

MANNAERTS, C. (1999): Factores de erosión, Curso de postgrado en levantamiento de recursos hídricos, Cochabamba, Bolivia.

PERLES ROSELLÓ, M.J. y CANTARERO PRADOS, F. (2010): “Problemas y retos en el análisis de los riesgos múltiples del territorio. Propuestas metodológicas para la elaboración de cartografías multi-peligros", Boletín de la Asociación de Geógrafos Españoles, 52, 245-71.

PERLES ROSELLÓ, M.J.; GALLEGOS REINA, A. y CANTARERO PRADOS, F. (2006): "Análisis del ajuste del área inundable obtenida mediante una evaluación integrada de la peligrosidad de inundación y peligros asociados", Baetica. Estudios de Arte, Geografía e Historia, 28, 527-45.

ROBREDO SÁNCHEZ, J.C. (1993): Erosión, E.T.S. Ingenieros de Montes, Universidad Politécnica de Madrid.

TÉMEZ, J.R. (1978): Cálculo hidrometeorológico de caudales máximos en pequeñas cuencas naturales, MOPU, Dirección General de Carreteras, 113 pp.

TÉMEZ, J.R. (1991): "Extended and improved Rational Method. Version of the Highways Administration of Spain", en Proc. XXIV Congress, vol A, 33-40.

USSCS (1972): “National Engineering Handbook", Sec 4, sup. A, en Hydrology, Soil Conservation Service.

WISCHMEIER, W.H.; JOHNSON, C.B. y CROSS B.V. (1971): “A soil erodibility nomograph for farmland and construction sites", Journal of soil and water conservation, 26, 189-92.

WISCHMEIER, W.H. y SMITH, D.D. (1958): "Rainfall energy and its relationship to soil loss", Trans. Amer. Geophys. Union, 39, 285-91. 